\title{
Suicidal Ideation in Elite Schools: A Test of the Interpersonal Theory and the Escape Theory of Suicide
}

\author{
Hélène Landrault, ${ }^{1,2,3}$ Nemat Jaafari, ${ }^{3}$ Mohamed Amine, ${ }^{1}$ Gabriel Malka, ${ }^{1}$ \\ Leila Selimbegović, ${ }^{2}$ and Armand Chatard ${ }^{2,3^{*}}$ \\ ${ }^{1}$ Laboratoire d'épidémiologie et de biostatistiques, Université Mohammed VI Polytechnique, Maroc \\ ${ }^{2}$ Centre National de la Recherche Scientifique, Université de Poitiers, France \\ ${ }^{3}$ Unité de recherche clinique Pierre Deniker, Centre Hospitalier Henri Laborit, Poitiers, France
}

\begin{abstract}
Objective: The present study compared the ability of two contemporary theories of suicidal behaviorthe interpersonal and escape theories of suicide - to predict suicidal ideation. The interpersonal theory proposes that the interaction of perceived burdensomeness and thwarted belongingness predicts suicidal ideation. The escape theory proposes that feelings of failure predict suicidal ideation and that escape motivation mediates this relationship. The present study intended to determine which of the two theories more successfully explains suicidal ideation. Method: A sample of 306 students from elite schools in Morocco (193 women, $M_{\text {age }}=21.21$ years, predominantly Muslims) completed a questionnaire assessing feelings of failure and escape motivation, perceived burdensomeness and thwarted belongingness, suicide ideation, and control variables. Results: In line with previous research, separate tests revealed support for the two theories. Interestingly, when entered simultaneously in a multiple regression analysis, the two frameworks explained a unique and cumulative part of the variance in suicidal ideation. Moreover, the effects remained significant after controlling for past suicide attempts, depression, hopelessness, and stress. Conclusions: The findings suggest that combining the interpersonal and escape theories of suicide could help better explain the emergence of suicidal ideation among college students.
\end{abstract}

Keywords: interpersonal theory, escape theory, suicide ideation, feelings of failure and escape motivation, perceived burdensomeness and thwarted belongingness

Open research data: The materials, dataset, and code for this study are publicly available on the Open Science Framework: https://osf.io/gfdcq/

* Correspondence: Armand Chatard, Université de Poitiers, CeRCA, CNRS 7295, MSHS, Bat A5, 5 rue Théodore Lefebvre, TSA 21103, 86073 Poitiers CEDEX, France. Email: armand.chatard@, univ-poitiers.fr

Suicide is a leading cause of death worldwide (World Health Organization, 2014). Because the causes of suicide are not yet fully understood (O'Connor \& Nock, 2014), it is important to test alternative theories regarding suicidal thoughts and behaviors to determine which one most successfully explains suicidal behavior (Tarsafi, Kalantarkousheh, \& Lester, 2015). Testing psychological theories of suicidal thoughts and behavior may also contribute to the development of efficient suicide interventions (Franklin, Huang, Fox, \& Ribeiro, 2018). Although several theories concerning suicidal ideation and behaviors have been proposed (for a review, see O'Connor \& Nock, 2014), the two dominant frameworks are the interpersonal theory of suicide (Joiner, 2005; Van Orden et al., 2010) and the escape theory of suicide (Baumeister, 1990). Research indicates that these two frameworks, studied in isolation, help explain a significant part of the variance in suicidal ideation. To date, however, no research has tested the predictive value of the two frameworks. Thus, their relative contribution to explaining suicidal ideation still remains unknown. The aim of the present study was to address this issue. Using a sample of students from elite schools in Morocco, we examined whether and how the two theoretical models predict suicidal ideation when tested simultaneously and concurrently.

The interpersonal theory of suicide (Joiner, 2005; Van Orden et al., 2010) stresses the importance of social bonds and attachment processes. According to this framework, suicidal ideation emerges from the simultaneous presence of two distinct but related interpersonal constructs: thwarted belongingness and perceived burdensomeness. Thwarted belongingness is a negative feeling about oneself that results when one's 
fundamental need for connectedness (Baumeister \& Leary, 1995) is not fulfilled. The feeling is usually assessed with items like "These days, I feel disconnected from other people" (Van Orden, Cukrowicz, Witte, \& Joiner, 2012). It has also been operationalized through various indices of social isolation, such as loneliness and low social support (Van Orden et al., 2012). Perceived burdensomeness, the second construct in the interpersonal theory of suicide, emerges when one perceives oneself as a burden to close others. It is usually assessed with items like "These days, the people in my life would be happier without me" (Van Orden et al., 2012). Perceived burdensomeness has also been examined with conceptually related constructs, such as family discord, unemployment, and functional impairment (Van Orden et al., 2012). Previous studies provide evidence that thwarted belongingness and perceived burdensomeness interact to predict suicidal ideation (for reviews, see Chu et al., 2017; Ma, Batterham, Calear, \& Han, 2016). When both are relatively high, one's desire for suicideand thus one's suicidal ideation-increases. The interpersonal theory of suicide further predicts that thwarted belongingness and perceived burdensomeness are necessary but not sufficient for a suicide attempt to occur; an individual with high levels of these two constructs will only attempt to end her life if she has surpassed the fear of death (described as the acquired capacity for suicide).

The escape theory of suicide (Baumeister, 1990) stresses the roles of failure, disappointment, and setback. According to this framework, suicidal ideation emerges when one is flooded with intense feelings of failure and suffers from negative affect, motivating one to escape this negative self-awareness. From this perspective, one comes to think about suicide because it appears as the only solution to one's problems. A comprehensive review of the literature provided support for this theory (Baumeister, 1990). More recently, a series of experimental studies confirmed the most basic predictions of escape theory (Chatard \& Selimbegović, 2011; Chatard, Selimbegović, Pyszczynski, \& Jaafari, 2017; Selimbegović \& Chatard, 2013; Tang, Wu, \& Miao, 2013). These studies indicate that when people realize their failure to attain high performance or value, suicidal thoughts become more accessible (Chatard \&
Selimbegović, 2011; Tang et al., 2013). Moreover, the desire to escape the self, exacerbated by seeing one's image in a mirror, can bring suicide to the fringe of consciousness (Selimbegović \& Chatard, 2013). Finally, this stream of literature shows that the increased accessibility of suicidal thoughts following contemplation about failure is especially pronounced when associated with negative affect (Chatard et al., 2017). From a theoretical viewpoint, the escape theory of suicide implies that feelings of failure should predict suicide ideation and that motivation to escape selfawareness should mediate the relation between failure and suicidal ideation. The escape theory further predicts that feelings of failure and motivation to escape are necessary, but not sufficient, for one to attempt suicide; this will happen only after an individual has undergone a six-step process of considering suicide and found no alternative means of escape. In addition to feelings of failure and escape motivation, this process includes self-blame, depression, cognitive deconstruction, and disinhibition.

Interestingly, the notion that failure and escape motivation are key determinants of suicidal behavior is central to other contemporary theories of suicide, such as the defeat-entrapment theory (Gilbert \& Allan, 1998; Taylor, Gooding, Wood, \& Tarrier, 2011; Taylor, Gooding, Wood, Johnson, \& Tarrier, 2011) and the integrated motivationalvolitional theory (O'Connor, 2011). Although there are important differences between these frameworks and the escape theory, all consider failure (or defeat) and escape motivation (or entrapment) to play a central role in suicidal ideation and behavior.

There is substantial body of evidence supporting the predictions of the interpersonal and escape theories of suicide. However, these theories' joint ability to predict suicidal ideation has never been scientifically tested. It is worth noting that, at the theoretical level, the two theories do not overlap. In fact, they depend on conceptually different psychological constructs; the interpersonal theory emphasizes the role of interpersonal relationships (thwarted belongingness and burdensomeness), whereas the escape theory emphasizes the role of negative life experiences (feelings of failure). Thus, the two models might help explain separate and cumulative parts of the variance in suicide ideation. The present study 
attempted to test this hypothesis for the first time.

In line with the interpersonal theory, we predicted that perceived burdensomeness and thwarted belongingness would interact to predict suicide ideation among college students. That is, suicide ideation should be especially common among students who report relatively high levels of both factors. In line with the escape theory, we hypothesized that feelings of failure should predict suicidal ideation and escape motivation should significantly mediate this relationship. As in previous research, we expected to find support for these hypotheses when the two theories were tested separately. Extending previous research, we further expected that the two theories would provide unique and independent predictions regarding suicidal ideation, when considered simultaneously.

The central thesis of this paper is that combining the interpersonal and escape theories of suicide could help better explain the emergence of suicidal ideation among college students. To identify the unique effects of each theory, we controlled for additional variables: past suicide attempts, depression, stress, and hopelessness. It was hypothesized that the two theories would help explain suicidal ideation after controlling for these ancillary measures.

\section{Method}

\section{Participants}

In total, 306 college students from top universities in Morocco (193 women, $M_{\text {age }}=$ 21.21 years, $S D=2.95$ ) participated in this study. They were recruited from preparatory classes at the medical school of a private university in the Marrakech-Safi region, located in the center of Morocco. The present study extends previous research by testing the interpersonal and escape theories in Morocco, an understudied and predominantly Muslim country in North Africa.

\section{Procedure}

Upon agreeing to participate in the study, the students completed a paper-andpencil version $(\mathrm{n}=138)$ or an online version of a questionnaire $(n=168)$. The questionnaire was administered in French. In Morocco, French is widely used in school and education. In addition to demographic questions, we measured thwarted belongingness, perceived burdensomeness, feelings of failure, escape motivations, suicidal behavior, suicidal ideation, depression, hopelessness, and stress. The different measures are described below.

Demographic questions. Participants indicated their education, age, and gender on the questionnaire.

Thwarted belongingness and
perceived burdensomeness.
Interpersonal Needs Questionnaire (INQ; Van Orden et al., 2012) was used in this study. It is a 15-item self-report questionnaire that assesses feelings of thwarted belongingness (9 items, e.g., "These days, I am close to other people"; Cronbach's alpha $=0.78$ ) and perceived burdensomeness (6 items, e.g., "These days, the people in my life would be better off if I were gone"; Cronbach's alpha = 0.90). Participants indicated their response to each item on a 7-point Likert scale ranging from 1 ("Not at all true for me") to 7 ("Very true for me"). Because the INQ was not available in French, we made a Frenchlanguage version of the instrument (https://osf.io/gfdcq/) using a translation-back translation procedure.

Feelings of failure and escape motivations. Feelings of failure were assessed with the defeat scale (Gilbert \& Allan, 1998), a 16 -item self-report measure that assesses one's sense of failure and loss (e.g., "I feel that I am a successful person"; Cronbach's alpha = 0.95). Escape motivation was assessed with the entrapment scale (Gilbert \& Allan, 1998), a 16-item self-report measure that assesses escape motivation triggered by internal feelings and thoughts or by external factors (e.g., "I would like to escape from my thoughts and feelings"; Cronbach's alpha $=0.95$ ). Participants indicated on a 5-point Likert scale the degree to which the items represented their current thoughts and feelings $(0=$ "Not at all like me," 4 = "Extremely like me"). Because the defeat and entrapment scales were not available in French, we made French-language versions of the scales (https://osf.io/gfdcq/) using a translation-back translation procedure.

Suicidal ideation and behavior. Suicidal ideation and behavior were assessed with relevant items taken from the Mini International Neuropsychiatric Interview for suicidality disorder (Lecrubier et al., 1997). Suicidal ideation was assessed with two items ("I have had thoughts of killing myself in the past month" and "I have had thought of suicide 
in the past month"), each answered "yes" or "no." Responses to the two items were strongly correlated $(r[306]=0.55, p<.001)$. Thus, we summed the responses to the two items to form a continuous index of suicidal ideation $(\min =0, \max =2)$. Suicidal behavior was assessed with the item "I have made a suicide attempt in the past month", answered yes (coded 1) or no (coded 0$)$. In the present sample, seven participants $(2.3 \%)$ reported a suicide attempt in the past month.

Depression. The Beck Depression Inventory II (BDI-II; Beck, Steer, \& Brown, 1996) was used in this study to measure depressive symptoms. The BDI-II consists of 13 items, and each response is assigned a score of 0-3. Higher scores represent a higher occurrence of symptoms associated with depression. In the present sample, the internal consistency of the scale was Cronbach's alpha $=0.92$. Because suicide ideation was our primary outcome in this study, we excluded the suicide item from the composite score of depression. Thus, the depression score used in our analyses was the sum of the 12 remaining items.

Hopelessness. The Beck Hopelessness Scale (BHS; Beck, Weissman, Lester, \& Trexler, 1974) was used. The BHS is a 20 -item true-false self-report scale that is intended to measure the degree of one's pessimism about the future. A representative item is "I might as well give up because there's nothing I can do to make things better for me" (binary coded: 0 $=$ no, $1=$ yes $)$. In the present sample, the scale had high internal consistency (Cronbach's alpha $=.82$ ).

Perceived stress. The perceived stress scale (Cohen, Kamarck, \& Mermelstein, 1983) was used in this study. This measure is a selfreported 10-item instrument designed to measure the degree to which situations in one's life are appraised as stressful (i.e., threatening, unpredictable, uncontrollable, or painful). A representative item is "In the last month, how often have you felt nervous and stressed?" Scores ranged from 0 (never) to 4 (very often). The internal consistency of the scale was satisfactory (Cronbach's alpha $=.89$ ).

\section{Results}

Table 1 presents the Pearson correlations between all the variables measured in this study. The bivariate correlations between the different variables were quite high, with correlation coefficients ranging from $0.20-0.83$ (for all, $p<.005$ ). Moreover, all variables were significantly correlated to suicidal ideation.

\section{Interpersonal Theory and Suicidal Ideation}

We first examined the predictive ability of the interpersonal theory of suicide. In a linear regression model, suicidal ideation was predicted based on perceived burdensomeness, thwarted belongingness, and the product term of the two. All variables were mean-centered. A significant interaction between perceived burdensomeness and thwarted belongingness would provide support for the interpersonal theory. The model accounted for $26 \%$ of the variance in suicide ideation $(F[1,301]=35.51$, $p<.001)$. The main effects of perceived burdensomeness $(B=0.092, S E=0.034, \beta=$ $0.215, t[301]=2.697, p<.008)$ and thwarted belongingness $(B=0.080, S E=0.026, \beta=$ $0.183, t[301]=3.080, p<.003)$ were significant. As predicted by the interpersonal theory, the interaction between perceived burdensomeness and thwarted belongingness also reached significance $(B=0.053, S E=$ $0.017, \beta=0.217, t[301]=3.059, p<.003)$. The higher participants scored on the two constructs, the higher their reported levels of suicidal ideation.

\section{Escape Theory and Suicidal Ideation}

Next, we tested the predictive ability of the escape theory of suicide. According to this theory, feelings of failure should predict suicidal ideation and escape motivation should mediate this relationship. To test this hypothesis, we conducted a mediation analysis (Baron \& Kenny, 1986). In a stepwise regression analysis, suicidal ideation was predicted based on feelings of failure (step 1) and escape motivation (step 2). The model included two predictors accounting for $32 \%$ of the variance in suicide ideation $(F[2,303]=$ $71.52, p<.001)$. Both feelings of failure $(B=$ $0.353, S E=0.031, \beta=0.548, t[304]=11.410$, $p<.001)$ and escape motivation $(B=0.138, S E$ $=0.045, \beta=0.263, t[303]=3.05, p<.003)$ significantly predicted suicide ideation. Consistent with the mediation model, the effect of feelings of failure was substantially reduced in the second step $(B=0.211, S E=0.056, \beta=$ $0.327, t[303]=3.79, p<.001)$ compared to the first step. As predicted by the escape theory, a Sobel test indicated that escape motivation 
mediated the influence of feelings of failure on suicidal ideation $(Z=3.046, S E=0.046, p<$ $.003)$.

\section{Interpersonal Theory, Escape Theory, and Suicidal Ideation}

The reported analyses provided support for the interpersonal and escape theories of suicide when they were considered independently of each other. In the following analysis, we jointly examined predictors from each of the two theories. In a multiple regression analysis, suicidal ideation was predicted by feelings of failure as well as perceived burdensomeness, thwarted belongingness, and their product term. In a subsequent step of the regression model, we included escape motivation. All variables included in the equation were mean-centered. The beta coefficients and $p$-values are presented in Table 2, which shows that we found support for both theories when the respective predictors were entered simultaneously in a regression analysis. In Model 1, the interaction between perceived burdensomeness and thwarted belongingness and the effect of feelings of failure significantly predicted suicidal ideation. In Model 2, escape motivations significantly predicted suicidal ideation. After controlling for escape motivations, the effect of feelings of failure was significantly reduced $(Z=2.831$, $S E=0.039, p<.005)$, but not the interaction term between perceived burdensomeness and thwarted belongingness.

\section{Control Variables}

As shown in Table 3, we found similar findings when suicidal behavior, depression, hopelessness, and stress were entered in the regression analysis to control for their effects on suicidal ideation.

\section{Discussion}

The aim of the present study was to determine whether two dominant theories of suicide have validity for predicting suicidal ideation in a sample of college students from elite schools in Morroco. The hypotheses were largely supported; we found evidence supporting predictions derived from both the interpersonal and escape theories when considered separately as well as simultaneously. In line with the interpersonal theory, a significant part of the variance in suicidal ideation was explained by the interaction between perceived burdensomeness and thwarted belongingness. This finding is consistent with the view that suicidal ideation in students at top universities emerges in part when they suffer from both perceived burdensomeness and thwarted belongingness. Overall, the findings are consistent with previous research on the interpersonal theory of suicide (Chu et al., 2017; Ma et al., 2016; Van Orden et al., 2012). This study is important because it is the first to suggest that perceived burdensomeness and thwarted belongingness interact to predict suicidal ideation beyond feelings of failure, escape motivations, stress, depression, hopelessness, and suicidal behavior.

In line with the escape theory, a significant and independent portion of the variance in suicidal ideation was explained by feelings of failure, an effect that was partly mediated by escape motivation. This result is consistent with previous correlational studies showing that feelings of failure (measured with defeat items) prospectively predicted changes in suicidal ideation over time (Taylor, Gooding, Wood, Johnson, et al., 2011). It is also consistent with experimental studies suggesting that failure-induced escape motivation triggers suicide ideation among college students (Chatard \& Selimbegović, 2011; Chatard et al., 2017; Selimbegović \& Chatard, 2013; Tang et al., 2013). The present findings are important because they support a mediation model that is central to the escape theory and other frameworks that make similar predictions, such as the defeat-entrapment theory (Gilbert \& Allan, 1998; Taylor, Gooding, Wood, \& Tarrier, 2011) and the integrated motivational-volitional theory (O'Connor, 2011). Somewhat surprisingly, this mediation model had not been tested until this study. This may be partly due to the ongoing debate about whether feelings of failure (measured with defeat items) and escape motivation (measured with entrapment items) are best defined as two distinct constructs or as a single construct (Forkmann, Teismann, Stenzel, Glaesmer, \& De Beurs, 2018; Taylor, Wood, Gooding, Johnson, \& Tarrier, 2009). Although no consensus has been reached, the most recent studies using advanced network analyses suggest that defeat and entrapment items belong to different dimensions (Forkmann et al., 2018). In line with this 
conceptualization, the results of the present study indicate that the link between feelings of failure and suicidal ideation is significantly reduced when escape motivation is included in the model. In other words, suicidal ideation seems to arise in college students when they are strongly motivated to escape intense, unbearable feelings of failure. Remarkably, this mediation effect was found independently of perceived burdensomeness, thwarted belongingness, and their interaction as well as the effects of relevant control variables (stress, depression, hopelessness, and suicidal behavior).

The main contribution of the present study is its evidence showing, for the first time, that the interpersonal and escape theories of suicide are not redundant. Even though the key predictors of the two theories are strongly correlated (Table 1), they account for separate and cumulative parts of the variance in suicidal ideation. Interestingly, including escape motivation in the model reduced the effect of feelings of failure on suicidal ideation (consistent with mediation), but not the interaction between perceived burdensomeness and thwarted belongingness. Therefore, the two theories are best considered as complementary rather than competitive; the interpersonal theory does not predict suicidal ideation better than the escape theory, and vice versa. Opening new avenues of research, the present findings suggest that combining the interpersonal and escape theories of suicide could help better explain the emergence of self-destructive thoughts among college students.

Despite these strengths, certain limitations of the current study should be noted. First, the sample was demographically homogenous, as it included only young adults from elite schools in Morocco. It could be argued that feelings of failure and thwarted belongingness are especially strong in this sample because success is crucial in elite schools and these students are often separated from their parents. To increase the external validity of the findings, this study should be replicated in a more demographically diverse sample (e.g., older adults, students at less prestigious schools). It would also be interesting to replicate the study in clinical populations, such as individuals suffering from major depression or bipolar disorder.
Second, the present study investigated only suicidal ideation, not suicidal behavior. Thus, it remains unclear whether a combination of the two theories also successfully predicts suicidal behavior. Future studies exploring this issue should include additional variables, as the two theories suggest that the transition from suicidal ideation to a suicide attempt involves a number of variables not investigated in the present study. Our study was restricted to suicidal ideation because the two theories can be easily contrasted using this phenomenon, which is often considered the primary determinant of suicide plans and attempts (e.g., Nock et al., 2008). Moreover, it is often difficult to study suicidal behavior in non-clinical populations, such as the one used in this study, given the relatively low prevalence of suicide attempts.

To conclude, the present study provides substantial support for two contemporary theories of suicide. It is the first study to demonstrate that the interpersonal and escape theories have additive (cumulative) rather than subtractive (suppressing) effects on the prediction of suicidal ideation. The present findings complement and extend suicide researchers' recent effort to compare different theories in different countries (Tarsafi et al., 2015). Given the very strong correlations between different predictors of suicide ideation, researchers should make systematic efforts to disentangle the relative contribution of each theory. The present study represents an important step towards this goal.

\section{References}

Baron, R. M., \& Kenny, D. A. (1986). The moderator-mediator variable distinction in social psychological research: Conceptual, strategic, and statistical considerations. Journal of Personality and Social Psychology, 51, 1173-1182.

Baumeister, R. F., \& Leary, M. R. (1995). The need to belong: Desire for interpersonal attachments as a fundamental human motivation. Psychological Bulletin, 117, 497-529.

Baumeister, R. F. (1990). Suicide as escape from self. Psychological Review, 97(1), 90-113.

Beck, A. T., Weissman, A., Lester, D., \& Trexler, L. (1974). The measurement of 
pessimism: The hopelessness scale. Journal of Consulting \& Clinical Psychology, 42, 861-865.

Beck, A. T., Steer, R. A., \& Brown, G. K. (1996). Beck depression inventory-II. San Antonio, 78(2), 490-498.

Chatard, A., \& Selimbegović, L. (2011). When self-destructive thoughts flash through the mind: Failure to meet standards affects the accessibility of suiciderelated thoughts. Journal of Personality and Social Psychology, 100(4), 587-605.

Chatard, A., Selimbegović, L., Pyszczynski, T., \& Jaafari, N. (2017). Dysphoria, failure, and suicide: Level of depressive symptoms moderates effects of failure on implicit thoughts of suicide and death. Journal of Social and Clinical Psychology, 36(1), 1-21.

Chu, C., Buchman-Schmitt, J. M., Stanley, I. H., Hom, M. A., Tucker, R. P., Hagan, C. R., ... \& Michaels, M. S. (2017). The interpersonal theory of suicide: A systematic review and meta-analysis of a decade of cross-national research. Psychological Bulletin, 143(12), 13131345.

Cohen, S., Kamarck, T., \& Mermelstein, R. (1983). A global measure of perceived stress. Journal of Health and Social Behavior, 24(4), 385-396.

Forkmann, T., Teismann, T., Stenzel, J. S., Glaesmer, H., \& De Beurs, D. (2018). Defeat and entrapment: More than meets the eye? Applying network analysis to estimate dimensions of highly correlated constructs. BMC Medical Research Methodology, 18(1), 16.

Franklin, J. C., Huang, X., Fox, K. R., \& Ribeiro, J. D. (2018). What suicide interventions should target. Current Opinion in Psychology, 22, 50-53.

Gilbert, P., \& Allan, S. (1998). The role of defeat and entrapment (arrested flight) in depression: An exploration of an evolutionary view. Psychological Medicine, 28(3), 585-598.

Joiner, T. (2005). Why people die by suicide. Cambridge, MA: Harvard University Press.

Lecrubier, Y., Sheehan, D. V., Weiller, E., Amorim, P., Bonora, I., Sheehan, K. H., ... \& Dunbar, G. C. (1997). The Mini International Neuropsychiatric Interview (MINI). A short diagnostic structured interview: reliability and validity according to the CIDI. European Psychiatry, 12(5), 224-231.

Ma, J., Batterham, P. J., Calear, A. L., \& Han, J. (2016). A systematic review of the predictions of the InterpersonalPsychological Theory of Suicidal Behavior. Clinical Psychology Review, 46, 34-45.

Nock, M. K., Borges, G., Bromet, E. J., Alonso, J., Angermeyer, M., Beautrais, A., ... \& De Graaf, R. (2008). Crossnational prevalence and risk factors for suicidal ideation, plans and attempts. The British Journal of Psychiatry, 192(2), 98-105.

O'Connor, R. C. (2011). Towards an integrated motivational-volitional model of suicidal behaviour. In O'Connor, R.C., Platt, S., \& Gordon, J. (Eds), International handbook of suicide prevention: Research, policy and practice (1st ed., pp. 181-198). Oxford: John Wiley \& Sons, Ltd.

O'Connor, R. C., \& Nock, M. K. (2014). The psychology of suicidal behaviour. The Lancet Psychiatry, 1(1), 73-85.

Selimbegović, L., \& Chatard, A. (2013). The mirror effect: Self-awareness alone increases suicide thought accessibility. Consciousness and Cognition, 22(3), 756-764.

Tang, J., Wu, S., \& Miao, D. (2013). Experimental test of escape theory: Accessibility to implicit suicidal mind. Suicide and Life-Threatening Behavior, 43(4), 347-355.

Tarsafi, M., Kalantarkousheh, S. M., \& Lester, D. (2015). The defeat-entrapment theory versus Beck's hopelessness theory of depression and suicidality: A crossnational analysis in Iran and the United States. International Journal of Social Psychiatry, 61(8), 777-780.

Taylor, P. J., Gooding, P., Wood, A. M., \& Tarrier, N. (2011). The role of defeat and entrapment in depression, anxiety, and suicide. Psychological Bulletin, 137(3), 391.

Taylor, P. J., Gooding, P. A., Wood, A. M., Johnson, J., \& Tarrier, N. (2011). Prospective predictors of suicidality: Defeat and entrapment lead to changes in suicidal ideation over time. Suicide 
and Life-Threatening Behavior, 41(3), 297-306.

Taylor, P. J., Wood, A. M., Gooding, P., Johnson, J., \& Tarrier, N. (2009). Are defeat and entrapment best defined as a single construct? Personality and Individual Differences, 47(7), 795-797.

Van Orden, K. A., Witte, T. K., Cukrowicz, K. C., Braithwaite, S. R., Selby, E. A., \& Joiner, T. E., Jr. (2010). The interpersonal theory of suicide. Psychological Review, 117(2), 575-600.
Van Orden, K. A., Cukrowicz, K. C., Witte, T. K., \& Joiner, T. E., Jr. (2012). Thwarted belongingness and perceived burdensomeness: Construct validity and psychometric properties of the Interpersonal Needs Questionnaire. Psychological Assessment, 24(1), 197215.

World Health Organization. (2014). Preventing suicide: a global imperative. Geneva: World Health Organization. 
Table 1

Pearson Correlations

\begin{tabular}{lrrrrrrrrr}
\hline & 1 & 2 & 3 & 4 & 5 & 6 & 7 & 8 & 9 \\
\hline 1. Burdensomeness & - & & & & & & & \\
2. Thwarted belongingness & 0.544 & - & & & & & & \\
3. Feelings of failure & 0.713 & 0.639 & - & & & & & \\
4. Escape motivations & 0.655 & 0.608 & 0.836 & - & & & & \\
5. Suicidal behavior & 0.384 & 0.208 & 0.259 & 0.222 & - & & & \\
6. Hopelessness & 0.571 & 0.492 & 0.721 & 0.655 & 0.238 & - & & \\
7. Depression & 0.690 & 0.597 & 0.842 & 0.819 & 0.257 & 0.731 & - & \\
8. Stress & 0.545 & 0.496 & 0.771 & 0.768 & 0.168 & 0.585 & 0.748 & - \\
9. Suicidal ideation & 0.468 & 0.371 & 0.548 & 0.537 & 0.251 & 0.516 & 0.514 & 0.415 & - \\
\hline
\end{tabular}

Note. All $p$-values are $<.005$. 
Table 2

Standardized and Unstandardized Coefficients

\begin{tabular}{rlrcccr}
\hline Model & $\mathbf{B}$ & $\mathbf{S E}$ & $\boldsymbol{\beta}$ & $\mathbf{t}$ & \multicolumn{1}{c}{$\mathbf{p}$} \\
\hline $1 \quad$ Intercept & 0.187 & 0.030 & & 6.220 & $<.001$ \\
& Burdensomeness & -0.007 & 0.036 & -0.016 & -0.192 & 0.848 \\
& Thwarted belongingness & 0.012 & 0.027 & 0.026 & 0.422 & 0.673 \\
& Burdensomeness X Thwarted belongingness & 0.053 & 0.017 & 0.216 & 3.226 & 0.001 \\
& Feelings of failure & 0.286 & 0.048 & 0.441 & 5.943 & $<.001$ \\
& & & & & $\mathrm{R}^{2}=.339$ \\
2 Intercept & 0.185 & 0.030 & & 6.248 & $<.001$ \\
& Burdensomeness & -0.019 & 0.036 & -0.045 & -0.529 & 0.597 \\
& Thwarted belongingness & -0.000 & 0.027 & -0.000 & -0.010 & 0.992 \\
& Burdensomeness X Thwarted belongingness & 0.055 & 0.016 & 0.222 & 3.350 & $<.001$ \\
& Feelings of failure & 0.174 & 0.061 & 0.269 & 2.838 & 0.005 \\
& Escape motivation & 0.130 & 0.045 & 0.247 & 2.859 & 0.005 \\
& & & & & $\mathrm{R}^{2}=.357$ \\
\hline
\end{tabular}


Table 3

Standardized and Unstandardized Coefficients

\begin{tabular}{lrrrrr}
\hline Model & B & SE & $\boldsymbol{\beta}$ & t & p \\
\hline Intercept & 0.195 & 0.030 & & 6.584 & $<.001$ \\
Suicidal behavior & 0.263 & 0.187 & 0.071 & 1.410 & 0.159 \\
Depression & -0.002 & 0.007 & -0.028 & -0.278 & 0.781 \\
Hopelessness & 0.026 & 0.010 & 0.181 & 2.533 & 0.012 \\
Stress & -0.008 & 0.006 & -0.109 & -1.378 & 0.169 \\
Burdensomeness & -0.026 & 0.037 & -0.061 & -0.711 & 0.478 \\
Thwarted belongingness & -0.006 & 0.027 & -0.013 & -0.205 & 0.838 \\
Burdensomeness X Thwarted belongingness & 0.044 & 0.017 & 0.180 & 2.648 & 0.009 \\
Feelings of failure & 0.154 & 0.071 & 0.238 & 2.172 & 0.031 \\
Escape motivation & 0.148 & 0.050 & 0.282 & 2.928 & 0.004 \\
& & & & & $\mathrm{R}^{2}=.380$ \\
\hline
\end{tabular}

\footnotetext{
Note. When all control variables were included in the model, the interaction between perceived burdensomeness and thwarted belongingness as well as the effects of feelings of failure and escape motivation were significant. Consistent with mediation, escape motivation mediated the influence of feelings of failure on suicidal ideation $(Z=2.529, S E=0.022, p<.012)$.
} 